\title{
Correction to: Model parameter estimation and its uncertainty for 2-D inclined sheet structure in self-potential data using crow search algorithm
}

\author{
Arif Haryono ${ }^{1,2}$ (D) Sungkono ${ }^{2} \cdot$ Reni Agustin $^{2} \cdot$ Bagus Jaya Santosa $^{2} \cdot$ \\ Amien Widodo ${ }^{3}$. Bramantya Ramadhany ${ }^{4}$
}

Published online: 11 January 2021

C Akadémiai Kiadó 2021

\section{Correction to: Acta Geodaetica et Geophysica (2020) 55:691-715 https://doi.org/10.1007/s40328-020-00321-5}

In subtitle "Tambakrejo SP anomaly, Indonesia", page 710, for describing Fig. 13 written: "Besides those two faults, the inversion data result also produce four other minor faults with different position (Fig. 13 lower panel)" should be revised as "Besides those two faults, the inversion data result also produce seven other minor faults with different position (Fig. 13 lower panel)".

The original article can be found online at https://doi.org/10.1007/s40328-020-00321-5.

Arif Haryono

arif.haryono@fmipa.unmul.ac.id

Sungkono

hening_1@physics.its.ac.id

1 Department of Physics, Universitas Mulawarman, Kampus Gunung Kelua, Samarinda, East Kalimantan, Indonesia

2 Department of Physics, Institut Teknologi Sepuluh Nopember, Kampus ITS Sukolilo, Surabaya, East Java, Indonesia

3 Department of Geophysical Engineering, Institut Teknologi Sepuluh Nopember, Kampus ITS Sukolilo, Surabaya, East Java, Indonesia

4 Regional Disaster Management Center, 55 S. Parman Street, Waru - Sidoarjo, East Java, Indonesia 